\title{
The Perspectives of Healthcare Professionals and Patients on the Value of Collaborative Care Model for Diabetes in Primary Healthcare Settings in Qatar
}

Sara Abdulrhim¹, Mohamed Izham Mohamed lbrahim¹, Sowndramalingam Sankaralingam¹, Mohammed Issam Diab", Mohamed Abdelazim Mohamed Hussain², Hend Al Raey², Mohammed Thahir Ismail², Ahmed Awaisu"

${ }^{1}$ Qatar University, College of Pharmacy; ${ }^{2}$ Qatar Petroleum Diabetes Clinic, Qatar
"Corresponding author: aawaisu@au,edu,aa

\section{Background}

- Diabetes is a complex chronic disease that cannot be adequately managed by a single healthcare profession ${ }^{1}$.

- Optimal diabetes management requires the valuable input of each member of the healthcare team 1.

Collaborative Care Model (CCM) assures the integration of knowledge, skills, values, and attitudes that aid team working within and among professions, and with patients and their families to improve health outcomes².

- The perceptions of CCM providers [i.e. healthcare professionals (HCPs)] and recipients (i.e. patients with diabetes) have been inadequately investigated and reported in the literature ${ }^{3}$

Therefore, we aim to evaluate the value of CCM in diabetes care at a primary healthcare $(\mathrm{PHC})$ setting in Qatar.

\section{Objectives}

I. To explore the perspectives of HCPs and patients regarding the value of $\mathrm{CCM}$ in diabetes management in PHC settings.

II. To determine the facilitators and the barriers to the optimal application of CCM in diabetes management in $\mathrm{PHC}$ settings.

\section{Methods}

- A purposive sampling strategy was used to include representative viewpoints from HCPs and patients at QPDC.

- A qualitative descriptive approach was used.

Semi-structured interviews.

- Deductive thematic analysis of the transcribed interviews.

\section{Data collection}

\section{Researcher reads and familiarize themselves with data}

\section{Researcher identifies coding units}

\section{Thematic data analysis}

\section{Results}

- Twenty-four interviews were conducted with HCPs and patients.

- Fourteen major themes revealed.

- The HCP group was multinational, including six Arab and six Asian HCPs.

- Patients had diabetes for an average of $8.7 \pm 8$ years. Half of the participants were male and employed.

- Generally, participants identified barriers and facilitators related to patients, HCPs, and healthcare system.

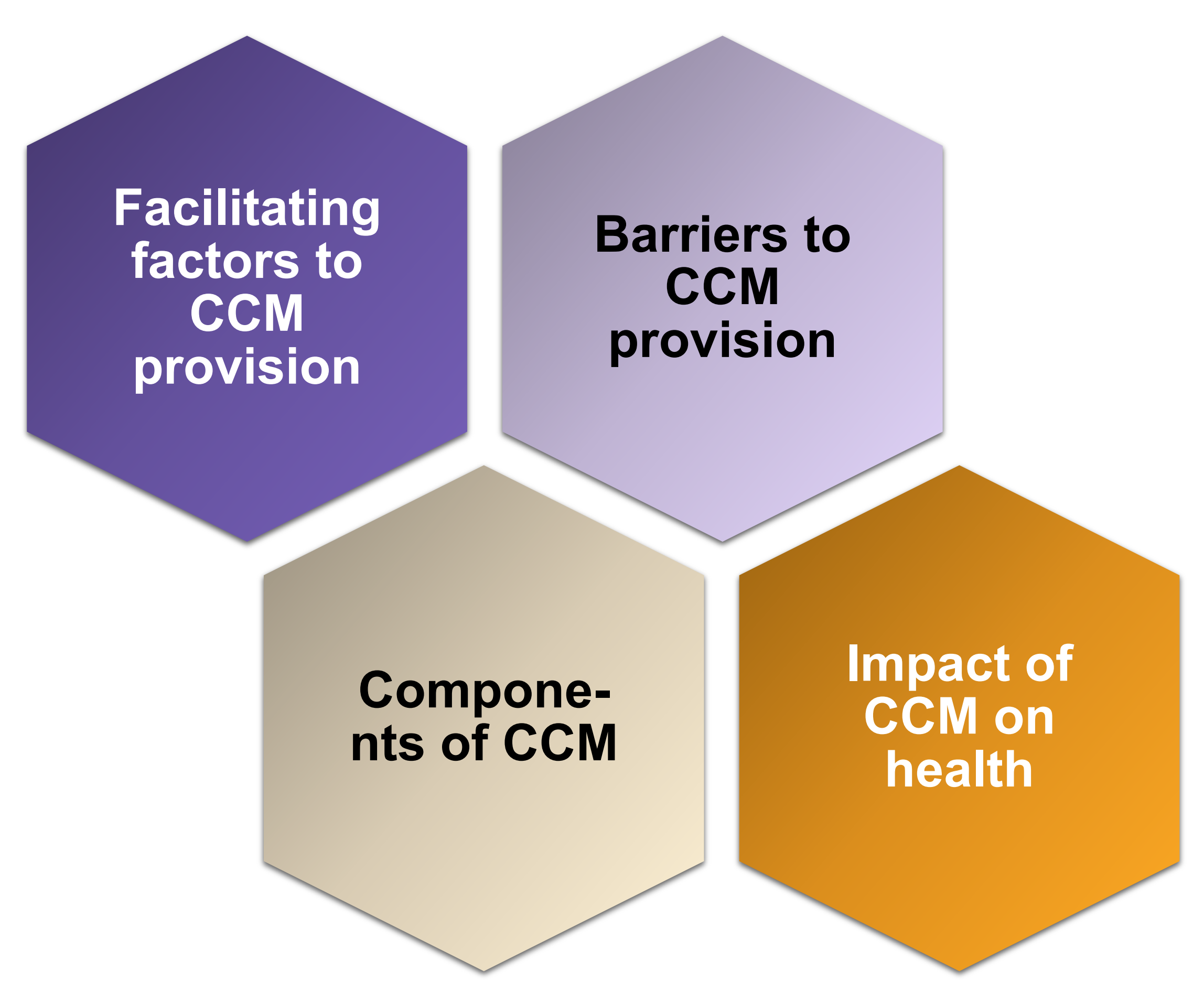

Figure 2. Study domains

- To facilitate collaborative practice in similar settings, pleasant attitudes of patients and HCPs besides administrative support through tangible resources must be considered.

"The doctor supervise the treatment plan, discuss, hear me, and talk friendly. The pharmacist doesn't refuse anything I ask for, he also discusses with me in a friendly language. The nurses are patient with us and educate us" (Patient 5).

\section{Theme 2: Barriers to CCM provision}

- Unpleasant attitude and undesirable attributes of HCPs and patients, unsupportive hospital system, and high workload were some of the identified barriers to CCM provision to patients with diabetes in $\mathrm{PHC}$ settings.

"Doctors would like to collaborate, but they have a time pressure. They don't have the chance to work with a team. They have a lot of patient load. We can make awareness for people regarding this issue" (Pharmacy manager).

\section{Results (Cont'd) \\ Theme 3: Components of CCM \\ - Important components of CCM such as HCPs' and patients' attributes and attitudes, family involvement in the care process, availability of technology, and utilization of facilities were recognized by the participants.}

"HCPs should have educational background, certified certificate from a well-recognized institute, experience. Because if you ask me about diabetes management 15 years ago it will completely be different from now" (Primary care physician).

\section{Theme 4: Impact of CCM on health}

- The provision of CCM may promote patients' health, level of patients and HCPs satisfaction, and preference of the service over other forms of care.

"I deal with patients with hypo- and hyperglycemia. Before the clinic, these incidents were very high. But after patients are followed-up in the clinic, they have less fluctuations in their blood glucose level... Now we rarely have emergency cases because of the good service patients receive at the clinic" (Nurse).

\section{Limitations}

There was an issue of anonymity and confidentiality while presenting the findings as the sample size is relatively small and participants could be identified by others through their demographics.

- Finally, the study is limited to one geographical location (i.e. Dukhan); nevertheless, the findings can be transferable to other similar settings.

\section{Conclusion}

The providers and users of CCM had an overall positive perception and appreciation of this model in $\mathrm{PHC}$ settings

Barriers to CCM such as unpleasant attitude and undesirable attributes of HCPs and patients, unsupportive hospital system, and high workload must be addressed before implementing the model in other PHC settings.

\section{References} Cheong LH, Armour CL, Bosnic-Anticevich SZ. Multidisciplinary collaboration in
primary care: Through the eyes of patients. Aust J Prim Health. 2013;19(3):190DeLoach C. The Impact of Interprofessional Collaboration on Diabetes Johnson JM. Carragher R. Interprofessional collaboration and the care and management of type 2 diabetic patients in the Middle East: A systematic review $J$ Interprof Care. 2018;32(5)::621-8. 